\title{
Body Mass Index and Mortality in Subjects With ARDS: Post-hoc Analysis of the OSCILLATE Trial
}

\author{
Haytham Tlayjeh, Yaseen M Arabi, Niall D Ferguson, Qi Zhou, Francois Lamontagne, \\ Alejandro Arroliga, Valerie Danesh, Guillermo Dominguez-Cherit, Edgar Jimenez, Adam Mullaly, \\ Brandon Staub, and Maureen O Meade; for the Canadian Critical Care Trials Group
}

BACKGROUND: Studies on the association of obesity with mortality in subjects with ARDS have yielded inconsistent results. METHODS: In a sub-analysis of the Oscillation for ARDS Treated Early (OSCILLATE) randomized controlled trial, 451 subjects were divided into 5 strata based on their body mass index (BMI) using the World Health Organization definitions: underweight $<18.5 \mathrm{~kg} / \mathrm{m}^{2}$; normal weight $18.5-24.99 \mathrm{~kg} / \mathrm{m}^{2}$; overweight $25-29.99 \mathrm{~kg} / \mathrm{m}^{2}$; obese $30-39.99 \mathrm{~kg} / \mathrm{m}^{2}$; severely obese $>40 \mathrm{~kg} / \mathrm{m}^{2}$. The primary outcome was all-cause hospital mortality across BMI strata for all subjects and for the 2 study arms (high-frequency oscillatory ventilation [HFOV] vs conventional ventilation) separately using multivariable logistic regression adjusting for potential confounding variables. RESULTS: Hospital mortality was not different across the BMI strata for all subjects $(P=.86)$, for the HFOV arm $(P=.94)$ or for the conventional ventilation arm $(P=.59)$. After risk adjustment, BMI was not associated with increased risk for hospital mortality (odds ratio 1.01, 95\% CI 0.97-1.04, $P=.67$ ), whereas HFOV was independently associated with increased mortality (odds ratio 1.74, 95\% CI 1.11$2.72, P=.02$ ) with no effect modification by BMI strata (for this interaction, $P=.56$ ). Although there was no difference in the use of rescue therapies or in the number of days on sedation or analgesia, higher daily doses of fentanyl and midazolam were administered as BMI increased. CONCLUSION: There was no difference in adjusted hospital mortality across BMI strata in subjects with moderate to severe ARDS. Processes of care were not different across BMI strata except for higher daily doses of fentanyl as BMI increased. (ClinicalTrials.gov registration NCT0150640) Key words: randomized; controlled trial; mechanical ventilation; high-frequency ventilation; obesity; mortality [Respir Care 2019;64(9):1042-1048. (C) 2019 Daedalus Enterprises]

\section{Introduction}

The prevalence of obesity in the world is increasing dramatically. Reports indicate that two thirds of the pop-

Drs Tlayjeh and Arabi are affiliated with the Intensive Care Department, King Saud bin Abdulaziz University for Health Sciences, King Abdullah International Medical Research Center, Riyadh, Saudi Arabia. Dr Ferguson is affiliated with the Interdepartmental Division of Critical Care Medicine, Departments of Medicine and Physiology, Institute for Health Policy, Management and Evaluation, Division of Respirology, University of Toronto, Toronto, Canada. Drs Zhou and Meade are affiliated with the Department of Health Research Methods, Evaluation and Impact, McMaster University, Hamilton, Canada. Dr Lamontagne is affiliated with Université de Sherbrooke, Sherbrooke, Canada and Centre de recherche du CHU de Sherbrooke, Sherbrooke, Canada. Dr Arroliga, Dr Jimenez, and Mr Mullaly are affiliated with the Department of Medicine, Baylor Scott and White Health, Temple, Texas. Dr Danesh is affiliated with the ulation in the United States is overweight or obese, with a body mass index $(\mathrm{BMI})>25 \mathrm{~kg} / \mathrm{m}^{2} .{ }^{1}$ Obese patients are at increased risk of comorbidities, have different adaptive mechanisms to illnesses and stresses, and altered cardiopulmonary physiology. Studies have demonstrated that hospitalized obese patients are more likely to require mechanical ventilation than non-obese patients. ${ }^{2}$ The association

\footnotetext{
School of Nursing, University of Texas at Austin, Texas. Dr DominguezCherit is affiliated with the Division of Pulmonary, Anesthesia and Critical Care Medicine, The National Institute of Medical Sciences and $\mathrm{Nu}-$ trition, Salvador Zubirán, Tlalpan, Mexico. Dr Staub is affiliated with the Department of Anesthesiology, University of Pittsburgh Medical Center, Pittsburgh, Pennsylvania.

The authors disclose a relationship with the Canadian Institutes of Health Research.
} 
between obesity and increased all-cause mortality in the general population is well described. ${ }^{3,4}$ Among critically ill patients, studies have shown that obesity is associated with an increased risk of developing ARDS, ${ }^{5}$ but the association between obesity and mortality in ARDS is inconsistent. ${ }^{6}$ A secondary analysis of the ARDS Network study comparing low tidal volumes of $6 \mathrm{~mL} / \mathrm{kg}$ versus

\section{See the Related Editorial on Page 1173}

$12 \mathrm{~mL} / \mathrm{kg}$ did not show any difference in outcome between obese and normal weight subjects. ${ }^{7}$ Other studies have suggested better survival for obese subjects with ARDS compared to subjects with normal weight. ${ }^{5}$ Studies have also shown that being underweight is associated with increased mortality in subjects with ARDS. ${ }^{8}$

The Oscillation for ARDS Treated Early (OSCILLATE) study was a randomized, controlled trial that randomized adults with moderate-to-severe ARDS to high-frequency oscillatory ventilation (HFOV) or conventional mechanical ventilation. The purpose of this sub-analysis of the OSCILLATE trial was to examine the association of BMI on mortality and relevant processes of care of subjects with moderate-to-severe ARDS. We hypothesized that mortality differs across different BMI strata and that these differences may be related to variations in certain processes of care. The OSCILLATE dataset represents a unique opportunity to address this question because it includes subjects who were managed closely by protocolized conventional ventilation that was based on lung-protective strategy. It also includes subjects managed with HFOV, a group in which the relation of BMI and outcomes has not been studied before.

\section{Methods}

\section{Study Subjects and Sample}

The OSCILLATE trial randomized 548 subjects with moderate-to-severe ARDS to HFOV or conventional ventilation from 39 ICUs in Canada, the United States, Saudi Arabia, Chile, and India. ${ }^{9}$ Institutional review boards of

Supplementary material related to this paper is available at http:// www.rcjournal.com.

Correspondence: Yaseen Arabi MD, Intensive Care Department, King Saud bin Abdulaziz University for Health Sciences, King Abdullah International Medical Research Center, King Abdulaziz Medical City, PO Box 22490, ICU 1425, Riyadh 11426, Saudi Arabia. E-mail: arabi@ngha.med.sa.

DOI: $10.4187 /$ respcare.06675

\section{QUICK LOOK}

\section{Current knowledge}

Obesity has been associated with increased risk of death in the general population. In ARDS, the association of obesity with mortality has yielded inconclusive results.

\section{What this paper contributes to our knowledge}

Death in hospital was not different among BMI strata in the whole study cohort, nor for the high-frequency oscillatory ventilation and conventional ventilation groups. High-frequency oscillatory ventilation was independently associated with increased mortality.

the participating centers approved the study. Patients were eligible for inclusion if they had had an onset of pulmonary symptoms within the previous 2 weeks, had undergone tracheal intubation, had hypoxemia $\left(\mathrm{P}_{\mathrm{aO}_{2}} / \mathrm{F}_{\mathrm{IO}_{2}} \leq 200\right.$, with an $\mathrm{F}_{\mathrm{IO}_{2}} \geq 0.5$ ), and had bilateral air-space opacities on chest radiography. Written informed consent was obtained from all the subjects or their legal representatives. After enrollment, a standardized assessment of hypoxemia was performed using pressure control mode, a tidal volume of $6 \mathrm{~mL} / \mathrm{kg}$, and an $\mathrm{F}_{\mathrm{IO}_{2}}$ of 0.60 with a PEEP level of $10 \mathrm{~cm} \mathrm{H} \mathrm{H}_{2} \mathrm{O}$ or higher. After $30 \mathrm{~min}$, if the $\mathrm{P}_{\mathrm{aO}_{2}} / \mathrm{F}_{\mathrm{IO}_{2}}$ ratio remained at or below 200, the subject was randomized. The trial excluded patients weighing $<35 \mathrm{~kg}$, obese patients with actual body weight $>1 \mathrm{~kg}$ per centimeter in height, and patients with documented chronic $\mathrm{CO}_{2}$ retention $\left(\mathrm{P}_{\mathrm{aCO}_{2}}>50 \mathrm{~mm} \mathrm{Hg}\right)$. For this secondary analysis, $451 / 548(82 \%)$ of subjects had available baseline weight data and were divided into 5 strata of BMI using baseline study data. The enrollment measured weight and height provided the data for BMI calculations for each subject. BMI was calculated by dividing subjects' body weight by the square of their height in meters $\left(\mathrm{kg} / \mathrm{m}^{2}\right) .{ }^{10}$ The strata were categorized based on the World Health Organization classification: ${ }^{10}$ underweight $<18.5 \mathrm{~kg} / \mathrm{m}^{2}$; normal weight 18.5-24.99 kg/m²; overweight $25-29.99 \mathrm{~kg} / \mathrm{m}^{2}$; obese $30-39.99 \mathrm{~kg} / \mathrm{m}^{2}$; severely obese $>40 \mathrm{~kg} / \mathrm{m}^{2}$.

Subject demographics, processes of care, and outcomes data were obtained from the original trial database. We extracted data on the following processes of care, which may affect outcome: the doses of intravenous infusions of sedatives and analgesics, the use of neuromuscular blockade, fluid balance (calculated as the average daily balance for the first $3 \mathrm{~d}$ ), and oxygenation rescue therapies (eg, nitric oxide, prone positioning, HFOV in the subjects in the conventional ventilation group). The primary outcome was hospital mortality. Secondary outcomes were ICU and 28-d mortality, mechanical ventilation settings, ICU and 
hospital length of stay (reported from the time of randomization), and incidence of barotrauma (pneumothorax, pneumomediastinum, pneumopericardium, or subcutaneous emphysema occurring spontaneously or after a recruitment maneuver).

\section{Statistical Analysis}

We reported categorical and continuous variables using means and standard deviation, medians and interquartile ranges, or counts and percentages, as appropriate. We compared demographic data, processes of care, and outcomes across BMI strata using chi-square and analysis of variance, as appropriate. We examined the independent association of BMI and hospital mortality by constructing a multivariate logistic regression model adjusting for the following variables: age (in 10-y increments), acute physiology score (in 5-unit increments), days in hospital prior to randomization, sepsis, and treatment group (HFOV vs conventional ventilation). Results are expressed as odds ratios (ORs) with 95\% CI. We implemented the above analyses for the whole cohort and for the 2 study arms (ie, HFOV and conventional ventilation) separately. To examine whether HFOV compared to conventional ventilation has a differential effect across BMI on mortality, we compared mortality between HFOV and conventional ventilation for each BMI strata. We tested whether BMI is an effect modifier of the association between the mode of ventilation and mortality by an interaction term in the model. Tests were 2 -sided, and a $P$ value $<.05$ was considered significant. SAS v. 9.3 (SAS Institute, Cary, North Carolina) was used to analyze the data.

\section{Results}

\section{Baseline Characteristics}

Table 1 shows the baseline characteristics of subjects across the BMI strata. There were no differences in the baseline characteristics of subjects by BMI strata for age, severity of illness, ARDS etiology, pre-enrollment ventilator settings, or severity of hypoxemia. Sex distribution was different across the BMI strata $(P=.005)$, with a higher proportion of females among the 2 highest BMI strata.

The pre-enrollment ventilator settings and the severity of hypoxemia were similar across the BMI strata (Table 1). Specifically, tidal volume, PEEP, plateau pressure, driving pressure, $\mathrm{P}_{\mathrm{aO}_{2}} / \mathrm{F}_{\mathrm{IO}_{2}}$, and $\mathrm{pH}$ were similar.

\section{Processes of Care}

Subjects received higher doses of midazolam and fentanyl as BMI increased (Table 2); however, there was no difference in the average daily dose of propofol or morphine. There was no difference in the number of days on sedation or analgesia or neuromuscular blockade, in cumulative fluid balance for the first $3 \mathrm{~d}$, or in the use of rescue therapies across BMI strata (Table 2). Process of care among subjects in the HFOV and conventional ventilation groups are shown in supplemental Table 2 (see the supplementary materials at http://www.rcjournal. com).

\section{Outcomes}

Death in hospital was not different among BMI strata in the whole study cohort (Fig. 1, Table 3). Running the analysis separately by group did not materially change any associations (Table 3 in the supplementary materials at http://www.rcjournal.com). After risk adjustment, BMI was not associated with increased risk for hospital mortality (OR 1.01, 95\% CI 0.97-1.04, $P=.67$ ), whereas HFOV was independently associated with increased mortality (OR 1.74, 95\% CI 1.11-2.72, $P=.02$ ) (Table 4). The association between HFOV and hospital mortality was not modified by BMI strata $(P$ value for interaction $=.56$, Fig. 1). There was no difference in mechanical ventilator days, ICU length of stay, or barotrauma across all BMI strata (Table 3).

\section{Discussion}

In the OSCILLATE trial, there was no difference in hospital mortality across BMI strata. BMI was not an effect modifier of the association between mode of ventilation and mortality. Subjects received higher doses of midazolam and fentanyl with increasing BMI strata, but this did not appear to translate to better or worse outcomes for increasing BMI levels.

Excess body weight has been associated with increased risk of developing ARDS. In a prospective study of 1,795 ICU subjects at risk for ARDS, obesity was associated with ARDS compared with normal weight (OR 1.66, 95\% CI 1.21-2.28 for obese; OR 1.78, 95\% CI 1.12-2.92 for severely obese). ${ }^{11}$ However, the effect of BMI on survival outcomes in subjects with ARDS is not clear.5,9 O'Brien et $\mathrm{al}^{6}$ examined the outcome of subjects enrolled in the ARMA trial and reported that the adjusted 28-d mortality was not affected by BMI (adjusted OR 0.80, $95 \%$ CI $0.30-2.13, P>.2$ ), and the benefits of lower tidal volume were similar for all BMI categories. In a related prospective cohort study, the mortality of 825 subjects with ARDS was lower in the severely obese subjects (25.9\%) compared to subjects with normal weight. The adjusted mortality, however, was not significantly different between all BMI categories. ${ }^{5}$ In a large $(N=1,488$ subjects) retrospective ARDS database study, lower BMIs 
Table 1. The Baseline Characteristics of Subjects Across the BMI Strata Before Randomization

\begin{tabular}{|c|c|c|c|c|c|c|}
\hline & \multicolumn{5}{|c|}{ BMI Strata } & \multirow{2}{*}{$P$} \\
\hline & $\begin{array}{c}<18.5 \\
(n=14)\end{array}$ & $\begin{array}{l}18.5-24.99 \\
(n=118)\end{array}$ & $\begin{array}{l}25-29.99 \\
(n=146)\end{array}$ & $\begin{array}{l}30-39.99 \\
(n=137)\end{array}$ & $\begin{array}{c}\geq 40 \\
(n=36)\end{array}$ & \\
\hline Age, y & $55.9(44.3-73.8)$ & $52.2(42.4-65.9)$ & $55.4(43.3-64.8)$ & $56.9(47.6-67.1)$ & $57.4(39.0-63.8)$ & .38 \\
\hline Female sex & $5(35.7)$ & $53(44.9)$ & $44(30.1)$ & $65(47.5)$ & $21(58.3)$ & .005 \\
\hline APACHE II score & $31.5(24-36)$ & $28(24-35)$ & $28(24-34)$ & $29(24-33)$ & $28.5(23-33.5)$ & .82 \\
\hline Time in hospital prior to study, $\mathrm{d}$ & $4.5(2-12)$ & $3(1-6)$ & $3(1-5)$ & $2(1-6)$ & $1(1-4)$ & .02 \\
\hline $\begin{array}{l}\text { Duration of mechanical ventilation } \\
\text { prior to study, } d\end{array}$ & $2(2-3)$ & $1(1-3)$ & $1(1-3)$ & $2(1-3)$ & $1(1-2.5)$ & .55 \\
\hline \multicolumn{7}{|l|}{ Risk factors for ARDS } \\
\hline Sepsis & $8(57.1)$ & $52(44.1)$ & $66(45.2)$ & $62(45.3)$ & $21(58.3$ & .54 \\
\hline Pneumonia & $6(42.9)$ & $76(64.4)$ & $88(60.3)$ & $92(67.2)$ & $17(47.2)$ & .11 \\
\hline Gastric aspiration & $4(28.6)$ & $24(20.3)$ & $22(15.1)$ & $17(12.4)$ & $6(16.7)$ & .29 \\
\hline Trauma & $0(0)$ & $2(1.7)$ & $5(3.4)$ & $7(5.1)$ & $0(0)$ & .51 \\
\hline H1N1 & $0(0)$ & $4(3.4)$ & $10(6.9)$ & $5(3.7)$ & $1(2.8)$ & .66 \\
\hline Other & $2(14.3)$ & $23(19.5)$ & $25(17.1)$ & $24(17.5)$ & $7(19.4)$ & .98 \\
\hline Ventilator mode on enrollment & & & & & & .16 \\
\hline Pressure assist control & $9(64.3)$ & 49 (41.5) & $84(57.5)$ & $71(51.8)$ & $22(61.1)$ & NA \\
\hline Volume assist control & $5(35.7)$ & $35(29.7)$ & $32(21.9)$ & $34(24.8)$ & $5(13.9)$ & NA \\
\hline Volume-targeted pressure control & $0(0)$ & $21(17.8)$ & $16(10.9)$ & $25(18.3)$ & $5(13.9)$ & NA \\
\hline Pressure support & $0(0)$ & $12(10.2)$ & $11(7.5)$ & $7(5.1)$ & $4(11.1)$ & NA \\
\hline Other & $0(0)$ & $1(0.9)$ & $3(2.1)$ & $0(0)$ & $0(0)$ & NA \\
\hline $\begin{array}{l}\text { Tidal volume, } \mathrm{mL} / \mathrm{kg} \text { predicted } \\
\text { body weight }\end{array}$ & $5.9(5.5-6.5)$ & $6.17(5.9-6.9)$ & $6.14(5.9-6.9)$ & $6.14(5.9-7.4)$ & $6.25(5.66-7.5)$ & .38 \\
\hline Plateau pressure, $\mathrm{cm} \mathrm{H}_{2} \mathrm{O}$ & $32(28-35)$ & $28.5(25-33)$ & $30(24-34)$ & $30(26-34)$ & $30(28-34)$ & .41 \\
\hline Set PEEP, $\mathrm{cm} \mathrm{H}_{2} \mathrm{O}$ & $13(10-15)$ & $12(10-15)$ & $12(10-15)$ & $14(10-16)$ & $15(12-17.5)$ & .09 \\
\hline Driving pressure, $\mathrm{cm} \mathrm{H}_{2} \mathrm{O}$ & $18(15-23)$ & $15(12-19)$ & $16(12-20)$ & $16(12-20)$ & $15(12,20)$ & .60 \\
\hline Minute ventilation, $\mathrm{L} / \mathrm{min}$ & $11.8(9.3-13.3)$ & $10.04(8.4-13.1)$ & $11(8.8-12.3)$ & $10.8(9.2-13)$ & $10.89(8.8-12.4)$ & .54 \\
\hline Oxygenation index & $15.6(14.1-29.3)$ & $17.48(11.3-25.8)$ & $16.5(11.8-23.6)$ & $19.91(12.5-25.9)$ & $21.76(16.8-25.7)$ & .11 \\
\hline $\mathrm{P}_{\mathrm{aO}_{2}} / \mathrm{F}_{\mathrm{IO}_{2}}$ & $125.2(109.3-150)$ & $108.33(78.8-141.7)$ & $120.83(93.5-148.1)$ & $109.17(82-136.7)$ & $110(86-125.3)$ & .19 \\
\hline $\mathrm{P}_{\mathrm{aCO}_{2}}, \mathrm{~mm} \mathrm{Hg}$ & $42(40-46)$ & $45(39-53)$ & $44(38.5-52)$ & $44(40-53)$ & $44(36.5-54.5)$ & .98 \\
\hline Arterial $\mathrm{pH}$ & $7.3(7.3-7.4)$ & $7.31(7.2-7.4)$ & $7.31(7.3-7.4)$ & $7.33(7.3-7.4)$ & $7.32(7.3-7.4)$ & .25 \\
\hline Use of HFOV & $8(57.1)$ & $65(55.1)$ & $79(54.1)$ & $73(53.3)$ & $16(44.4)$ & .84 \\
\hline
\end{tabular}

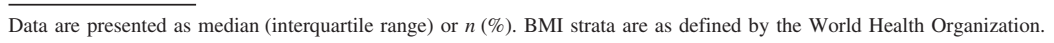

$\mathrm{BMI}=$ body mass index

APACHE II $=$ Acute Physiology and Chronic Health Evaluation

$\mathrm{HFOV}=$ high-frequency oscillatory ventilation

were associated with higher odds of death, whereas overweight and obese BMIs were associated with lower odds (overweight adjusted OR 0.72, 95\% CI 0.51-1.02; obese adjusted OR $0.67,95 \%$ CI $0.46-0.97$; severely obese adjusted OR 0.78, 95\% CI 0.44-1.38). ${ }^{2}$

We considered several explanations as to why the outcomes of patients with ARDS could differ across BMI strata and why studies have yielded inconsistent results regarding this association. First, there may be variations in case identifications across BMI strata, especially in nonprotocolized observational studies. For instance, very high BMI can confound chest radiograph interpretations with the appearance of lower lung volumes and thus can potentially lead to an overestimate of the extent of air-space disease. This is less likely in the OSCILLATE trial because the identification of cases was protocolized and included a standardized hypoxemia assessment. Second, very high BMI can confound estimates of disturbance in oxygenation as a result of effects on transpulmonary pressure.
Third, differences in mortality between obese and nonobese patients reported in some studies may be explained by other residual confounding ventilator parameters. ${ }^{7}$ However, this was not the case in our study because tidal volumes were not different across different BMI strata. ${ }^{12}$ Fourth, our study showed that obese subjects received higher doses of midazolam and fentanyl. Excessive use of sedation has been linked to poorer outcome in critically ill patients. This is further cofounded by the fact that most of these drugs are lipophilic ${ }^{13}$ and their volume of distribution is increased in obese individuals, further increasing the possibility of prolonged and excessive sedation in obese patients with ARDS ${ }^{14,15}$; however, we did not observe longer ICU or hospital lengths of stay among subjects with higher BMI. Higher doses of sedation may have facilitated lung-protective strategies and decreased risk of ventilatorinduced lung injury from patient-ventilator asynchrony by avoiding large swings of pleural pressure and thus high tidal volumes. ${ }^{16}$ Fifth, other aspects of general ICU man- 
BMI AND MoRTALITY IN ARDS

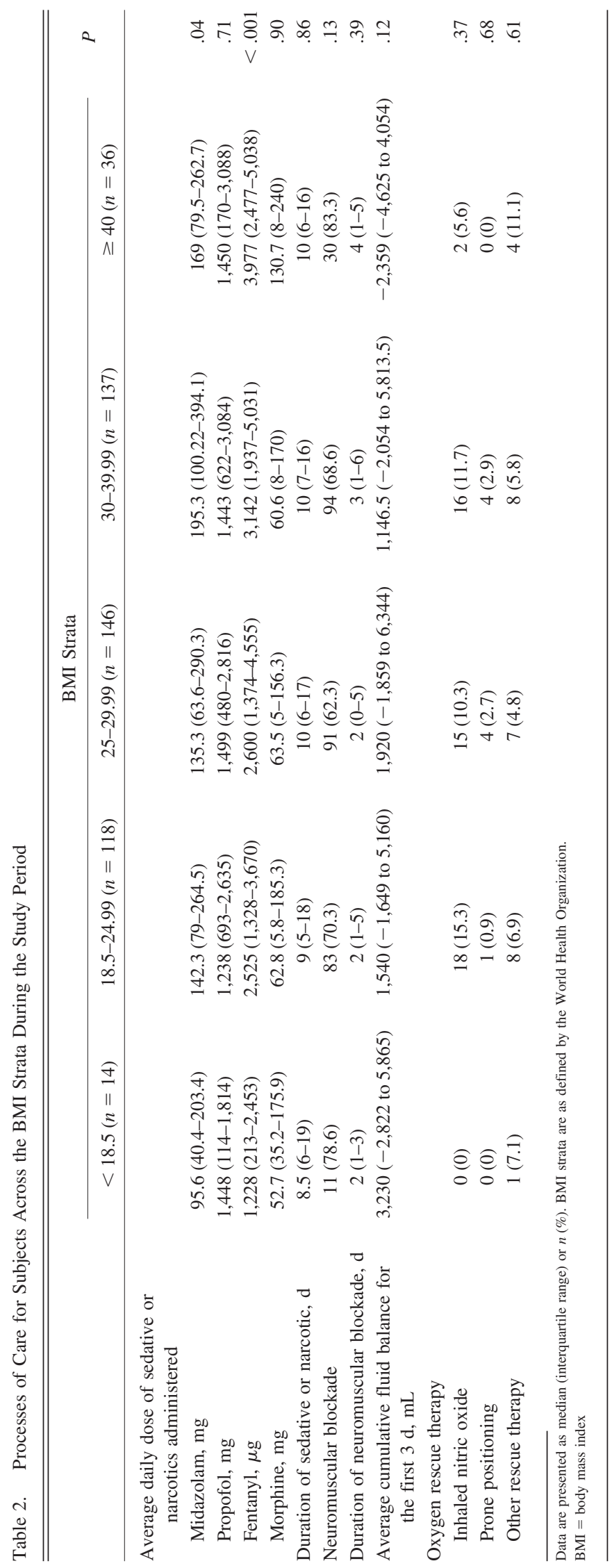



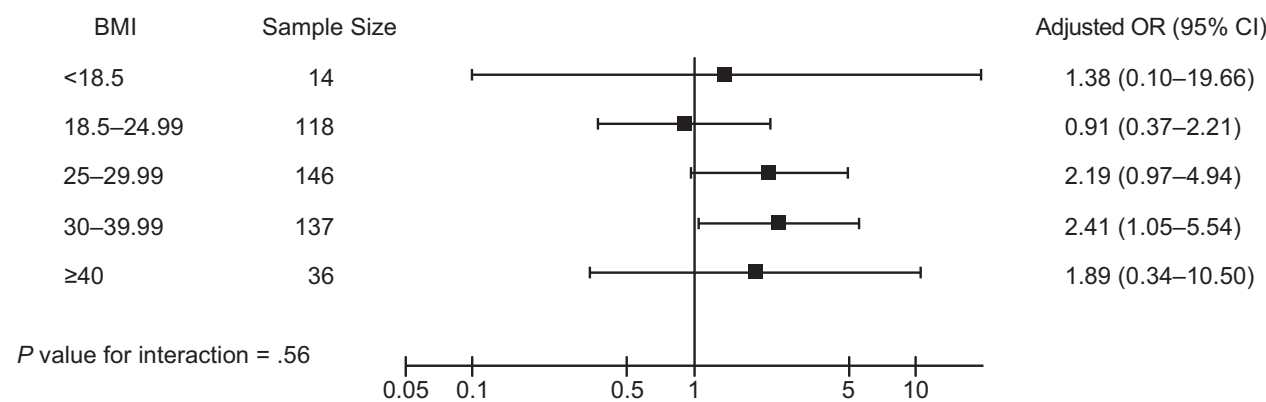

Fig. 1. In-hospital death according to BMI. BMI = body mass index; OR = odds ratio.

Table 3. Outcomes of Subjects Across the BMI Strata

\begin{tabular}{|c|c|c|c|c|c|c|}
\hline & \multicolumn{5}{|c|}{ BMI Strata } & \multirow[b]{2}{*}{$P$} \\
\hline & $\begin{array}{c}<18.5 \\
(n=14)\end{array}$ & $\begin{array}{c}18.5-24.99 \\
(n=118)\end{array}$ & $\begin{array}{l}25-29.99 \\
(n=146)\end{array}$ & $\begin{array}{l}30-39.99 \\
(n=137)\end{array}$ & $\begin{array}{c}\geq 40 \\
(n=36)\end{array}$ & \\
\hline Death in hospital & $6(42.9)$ & $51(43.2)$ & $57(39.0)$ & $56(40.9)$ & $12(33.3)$ & .86 \\
\hline Death in ICU & $6(42.9)$ & $46(39.0)$ & $53(36.3)$ & $50(36.5)$ & $12(33.3)$ & .96 \\
\hline New barotrauma & $4(28.6)$ & $28(23.7)$ & $29(19.9)$ & $20(14.6)$ & $5(13.9)$ & .28 \\
\hline New tracheostomy & $5(35.7)$ & $30(25.4)$ & $35(24.0)$ & $28(20.4)$ & $4(11.1)$ & .25 \\
\hline Refractory hypoxemia & $1(7.1)$ & $22(18.6)$ & $13(8.9)$ & $18(13.1)$ & $2(5.6)$ & .12 \\
\hline Refractory acidosis & $0(0)$ & $3(2.5)$ & $6(4.1)$ & $5(3.7)$ & $2(5.6)$ & .85 \\
\hline Duration of mechanical ventilation, $d$ & $7(6-14.5)$ & $10(5-19)$ & $11(7-17)$ & $13(8-20)$ & $9(7-1)$ & .29 \\
\hline Duration of mechanical ventilation in survivors, $d$ & $7(6-14.5)$ & $10(5-18.5)$ & $11(7-17)$ & $12.5(8-22)$ & $9(7-14.5)$ & .24 \\
\hline ICU length of stay, $d$ & $16(6-33)$ & $13(6-26)$ & $13(7-21)$ & $13(9-22)$ & $12(9-17)$ & .79 \\
\hline ICU length of stay in survivors, $d$ & $16(7.5-31.5)$ & $15(7.5-26)$ & $14(9-24)$ & $16(11-28)$ & $11.5(9-18.5)$ & .38 \\
\hline Hospital length of stay, $d$ & $27(16.5-63.5)$ & $24.5(15-42)$ & $30(14-41)$ & $30(16-48)$ & $22(15-31)$ & .58 \\
\hline Hospital length of stay in survivors, d & $27(16.5-63.5)$ & $23(14-40)$ & $29(14-40)$ & $30(16-45)$ & $22(15-31)$ & .51 \\
\hline \multicolumn{7}{|c|}{$\begin{array}{l}\text { Data are presented as median (interquartile range) or } n(\%) \text {. BMI strata are as defined by the World Health Organization. } \\
\text { New barotrauma }=\text { pneumothorax, pneumomediastinum, pneumopericardium, or subcutaneous emphysema occurring spontaneously or after a recruitment maneuver. Excluded from this category were } \\
\text { patients who had barotrauma at baseline. }\end{array}$} \\
\hline
\end{tabular}

agement may be different across different BMI strata. In an observational study, obese subjects were more likely to receive pharmacologic prophylaxis for thromboembolism. ${ }^{5}$ Finally, the extent of lung-protective strategies may be different across different BMI strata. In our study, subjects had similar plateau pressures across all BMI strata, but because obese patients tend to have lower transpulmonary pressures because of higher pleural pressure, they may have had additional lung protection or, to the contrary, they may have had more atelectotrauma from cyclic opening and closure of the alveolar units. There was no measurement of the pleural pressure by esophageal probes in our subjects. Offsetting this phenomenon, excessive use narcotics and midazolam in obese subjects might have negated any benefit that could have resulted from lower transpulmonary pressure and negative fluid balance.

Obesity affects chest wall mechanics; therefore, ventilation strategies may lead to different outcomes in obese subjects compared to non-obese subjects. ${ }^{17} \mathrm{HFOV}$, which delivers high mean airway pressure with small tidal volumes at very high frequencies has been recently examined in the OSCILLATE trial, which demonstrated that HFOV was associated with increased risk of death. This subanalysis indicates that the association of ventilation strategy and mortality was not modified by BMI strata. This is consistent with the findings of a recent individual-subject meta-analysis. ${ }^{18}$

Strengths of our study include prospective data collection, protocolized mechanical ventilation, and multicenter involvement. Unlike observational reports, the OSCILLATE trial included explicit clinical protocols that help reduce provider bias and practice variation in each BMI strata. Subjects in the trial had similar tidal volumes upon enrollment. Because outcomes may be different at both extremes of weight, we chose to categorize subjects according to BMI groups rather than use BMI as a con- 
Table 4. Logistic Regression Adjusted Analysis of Hospital Mortality With BMI

\begin{tabular}{lcc}
\hline \hline & $\begin{array}{c}\text { Odds Ratio } \\
(95 \% \text { CI })\end{array}$ & $P$ \\
\hline Age, 10-y increase & $1.40(1.21-1.64)$ & $<.001$ \\
Acute physiology score, 5-unit increase & $1.66(1.37-2.01)$ & $<.001$ \\
Time in hospital prior to randomization, d & $1.10(1.05-1.16)$ & $<.001$ \\
Sepsis, yes vs. no & $0.85(0.46-1.61)$ & .64 \\
BMI & $1.01(0.97-1.04)$ & .67 \\
HFOV versus conventional ventilation & $1.74(1.11-2.72)$ & .02
\end{tabular}

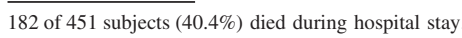

$\mathrm{BMI}=$ body mass index

$\mathrm{HFOV}=$ high-frequency oscillation ventilation

tinuous variable. Weaknesses include being a post hoc analysis. The OSCILLATE trial excluded obese patients with actual body weight $>1 \mathrm{~kg}$ per centimeter in height, which means that extremely obese patients are underrepresented in our cohort. In addition, we included only $82 \%$ of the OSCILLATE cohort because weight data were not available. Because the cohort was stratified into 5 strata, some of the analyses may have been underpowered, and true differences may not have been detected. Some studies have suggested that the association of mortality with BMI may follow a J-shaped or U-shaped curve, with increasing mortality in severe obesity and in very low BMI. Because the OSCILLATE trial excluded both extremes of weight, our study is not designed to address the associations at both extremes of BMI.

\section{Conclusions}

In the OSCILLATE Trial, there was no difference in adjusted hospital mortality across all BMI strata in subjects with moderate-to-severe ARDS. Processes of care were not different between the BMI strata except for higher daily doses of fentanyl as BMI increased.

\section{ACKNOWLEDGMENTS}

We acknowledge Michael Chasse and Martin Albert for their critical review of the manuscript.

\section{REFERENCES}

1. Ogden CL, Carroll MD, Kit BK, Flegal KM. Prevalence of childhood and adult obesity in the United States, 2011-2012. JAMA 2014; 311(8):806-814.
2. Kumar G, Majumdar T, Jacobs ER, Danesh V, Dagar G, Deshmukh A, et al. Outcomes of morbidly obese patients receiving invasive mechanical ventilation: a nationwide analysis. Chest 2013;144(1): 48-54.

3. Vaughan RW, Conahan TJ 3rd. Part I. cardiopulmonary consequences of morbid obesity. Life Sci 1980;26(25):2119-2127.

4. Anzueto A, Frutos-Vivar F, Esteban A, Bensalami N, Marks D, Raymondos K, et al. Influence of body mass index on outcome of the mechanically ventilated patients. Thorax 2011;66(1):66-73.

5. Morris AE, Stapleton RD, Rubenfeld GD, Hudson LD, Caldwell E, Steinberg KP. The association between body mass index and clinical outcomes in acute lung injury. Chest 2007;131(2):342-348.

6. O'Brien JM Jr, Welsh CH, Fish RH, Ancukiewicz M, Kramer AM, National Heart, Lung, and Blood Institute Acute Respiratory Distress Syndrome Network. Excess body weight is not independently associated with outcome in mechanically ventilated patients with acute lung injury. Ann Intern Med 2004;140(5):338-345.

7. Pelosi P, Croci M, Ravagnan I, Vicardi P, Gattinoni L. Total respiratory system, lung, and chest wall mechanics in sedated-paralyzed postoperative morbidly obese patients. Chest 1996;109(1):144-151.

8. Ni YN, Luo J, Yu H, Wang YW, Hu YH, Liu D, et al. Can body mass index predict clinical outcomes for patients with acute lung injury/acute respiratory distress syndrome? A meta-analysis. Crit Care 2017;21(1):36.

9. Ferguson ND, Cook DJ, Guyatt GH, Mehta S, Hand L, Austin P, et al. High-frequency oscillation in early acute respiratory distress syndrome. N Engl J Med 2013;368(9):795-805.

10. O'Brien JM Jr, Phillips GS, Ali NA, Lucarelli M, Marsh CB, Lemeshow S. Body mass index is independently associated with hospital mortality in mechanically ventilated adults with acute lung injury. Crit Care Med 2006;34(3):738-744.

11. Soubani AO, Chen W, Jang H. The outcome of acute respiratory distress syndrome in relation to body mass index and diabetes mellitus. Heart Lung 2015;44(5):441-447.

12. Arabi YM, Dara SI, Tamim HM, Rishu AH, Bouchama A, Khedr MK, et al. Clinical characteristics, sepsis interventions and outcomes in the obese patients with septic shock: an international multicenter cohort study. Crit Care 2013;17(2):R72.

13. Casati A, Putzu M. Anesthesia in the obese patient: pharmacokinetic considerations. J Clin Anesth 2005;17(2):134-145.

14. Cheymol G. Clinical pharmacokinetics of drugs in obesity. An update. Clin Pharmacokinet 1993;25(2):103-114.

15. Gong MN, Bajwa EK, Thompson BT, Christiani DC. Body mass index is associated with the development of acute respiratory distress syndrome. Thorax 2010;65(1):44-50.

16. The International Council for Harmonisation of Technical Requirements for Pharmaceuticals for Human Use (ICH). ICH Harmonised Tripartite Guideline. http://www.ich.org/fileadmin/ Public_Web_Site/ICH_Products/Guidelines/Efficacy/E9/Step4/ E9_Guideline.pdf. Accessed October 29, 2017.

17. Biring MS, Lewis MI, Liu JT, Mohsenifar Z. Pulmonary physiologic changes of morbid obesity. Am J Med Sci 1999;318(5):293-297.

18. Meade MO, Young D, Hanna S, Zhou Q, Bachman TE, Bollen C, et al. Severity of hypoxemia and effect of high-frequency oscillatory ventilation in acute respiratory distress syndrome. Am J Respir Crit Care Med 2017;196(6):727-733.

This article is approved for Continuing Respiratory Care Education credit. For information and to obtain your CRCE

(free to AARC members) visit www.rcjournal.com

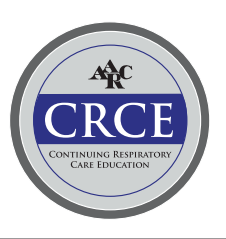

